\title{
A Jerk-constrained Asymmetric Motion Profile for High-speed Motion Stages to Reduce Residual Vibration
}

\author{
Huaizhong Li \\ School of Mechanical and Manufacturing Engineering, \\ The University of New South Wales, Sydney, NSW 2052, Australia \\ Email: hz.li@unsw.edu.au; lihuaizhong@gmail.com \\ Tel: +61-2-9385 5587; Fax: +61-2-9663 1222
}

\begin{abstract}
Control of the motion induced structural vibration is very important for high-speed and high-precision positioning stages. This paper presents a motion profile generation methodology to minimize jerks and residual vibrations for high-speed motion stages in precision manufacturing machines. Acceleration profile is smoothened by using sinusoidal functions. To reduce the residual vibration and settling time at the end point of the motion, the peak jerk constraint level at the deceleration phase is further decreased. In addition, the deceleration bound is also decreased as well. It results in an asymmetrical motion profile. The acceleration profile is used to derive a displacement profile, which can be implemented in a DSP-based motion controller to drive the motor. By using this motion profile, the residual vibration as well as settling time can be greatly reduced compared to traditional profiles like trapezoidal or S-curve profiles.
\end{abstract}

Keywords: motion control; residual vibration; positioning stage; jerk constraint; asymmetric motion profile

Biographical notes: Dr Huaizhong Li obtained a BE degree from Tsinghua University in 1988, ME degree from Xi' an Jiaotong University in 1991, and $\mathrm{PhD}$ degree from the National University of Singapore (NUS) in 2002, all in Mechanical Engineering. Dr Li has extensive research and industrial experience. He was an Engineer and project manager in Xi'an, China, from 1991 to 1998, and a software Engineer at GTECH Far East Pte Ltd, Singapore, in 2001. From 2001 to 2008, he was a Senior Research Engineer at Singapore Institute of Manufacturing Technologies (SIMTech), conducting research on advanced manufacturing technologies, machine dynamics, precision motion control and vibration control. He was an Associate Principal Engineer with Vestas Technology R\&D Singapore from 2008 to 2010, where his research focus was on wind turbine generator technologies to improve turbine reliability. He has been a lecturer in the School of Mechanical \& Manufacturing Engineering, the University of New South Wales, since 2011. His research interests and expertise span the areas of advanced manufacturing technologies, machine dynamics and vibration control, condition monitoring and advanced signal processing, etc.

\section{Introduction}

More and more ultra-precision manufacturing machines, such as ultra-precision machine tools, wafer steppers, nanotechnology process equipment, and micro CMMs, have been deployed in industry and research organisations in recent years. Vibration can lead to severe operational problems and limit their applications to achieve the required positioning accuracy and surface finish. Vibration in industry is caused by particular processes where dynamic forces excite structures. The sources of the disturbing vibrations can be building and floor vibrations, acoustic vibrations, or dynamic responses caused by the motorized equipment and machinery. The disturbing vibrations, if without efficient control, will lead to noise, wear, reduced accuracy and performance, etc. (Li et al, 2009; Bakar et al, 2011 and Liu et al, 2011).

For high-speed and high-precision positioning stages, control of the motion induced structural vibration is an important and also challenging task ( $\mathrm{Li}$ et al, 2009). Modern motion stages are characterised with increasing running 
speed and acceleration, higher accuracy, and more lightweight constructions. An aggressive motion profile will usually generate severe structural vibration and need long settling time. The settling time problem will be more critical when higher accuracy is required and the settling time window is tightened (Macfarlane and Croft, 2003). For example, the minimum feature size in today's semiconductor manufacturing is about $20 \mathrm{~nm}$, which requires a tighter allowable positioning error of only a few nanometers. On the other side, to satisfy the requirement of high throughput, the wafer lithography stage must run with high speed and high acceleration. It has been reported that the acceleration of modern wafer stages is up to $20-40 \mathrm{~m} / \mathrm{s}^{2}$, and the wafer scan speed during exposure is up to $0.6-1 \mathrm{~m} / \mathrm{s}$. As a result, the reaction force on the structure can be as large as 5,000-10,000 $\mathrm{N}$, which will generate significant dynamic excitations on the structure (Butler, 2011). In addition to optimising the structure design and employing active damping and active vibration isolation, advanced motion control strategies are also needed to minimise the dynamic excitation on the structure.

A typical point-to-point motion profile comprises phases of accelerating, constant speed, and decelerating. In a simple trapezoidal velocity profile, both the acceleration and deceleration are constant. It may cause some system disturbances at the corners of the velocity profile that translate into structural vibrations. The sudden change of acceleration will result in a significant jerk force to the structure of the motion stage. Hence it has the disadvantage of long settling time. Smoother motion profiles such as the s-curve velocity profile can be used to reduce the jerk force level. The velocity profile is modified to have an s-shape during the acceleration and deceleration periods to reduce the residual vibrations. It means that the trajectory ramps up to peak acceleration and ramps down to constant speed. However, it is noted that the s-curve profile still exhibits a sudden change in its jerk profile, and the finite jerk spreads out over a period of time (Li et al, 2009; Macfarlane and Croft, 2003).

To smooth the trajectory and bound jerk, higher order trajectory planning approaches have been investigated. A method using fourth-order feedforward with fourth-order trajectories for single-axis motion control was presented by Lambrechts et al. (2005). Macfarlane and Croft (2003) used a concatenation of fifth-order polynomial to provide a smooth trajectory for point-to-point motion with jerk limits. A sine wave template was used to calculate the end conditions for ramps from zero acceleration to nonzero acceleration in that approach. Li et al. (2009) proposed a low-vibration motion profile generation method to reduce the residual vibration. The acceleration profile was designed to have an s-shape by using a level-shifted sinusoidal waveform, which resulted in a smoother profile to cause little vibration at the motion end. Two high-performance point-to-point motion control algorithms have been presented by Wu et al. (2011). Some other research efforts on advanced motion control to reduce the motion induced vibration can be found in (Mahmood et al, 2008; Ha et al, 2011; Rew and Kim, 2010; and Hara, 2011).

It has been noted that a penalty for the smoother motion profile is a longer travel time for a given set of maximum acceleration and velocity ( $\mathrm{Li}$ et al, 2009). To enable the motion distance and motion time to be satisfied simultaneously, asymmetric velocity profiles with fixed motion time have been studied (Ha et al, 2008; Zou et al, 2011; Li et al, 2012; and Rew et al, 2009). Ha et al. (2008) proposed an asymmetric S-curve motion profile that enables easy manipulation of jerks during the arrival time in order to reduce the residual vibration. A design parameter of jerk ratio was introduced to scale down the jerks during the deceleration period so that the velocity profile is in an asymmetric S-curve. $\mathrm{Li}$ et al. (2012) developed an asymmetric velocity profile. By adjusting two scalars, the jerk in deceleration phase had a smaller value than it in acceleration phase. However, all the developed asymmetric velocity profiles are on the basis of conventional S-curve profile.

In this paper, the super S-curve profile presented in ( $\mathrm{Li}$ et al, 2009) is further developed into an asymmetrical motion profile, to improve the efficiency of reducing the residual vibration and the settling time, with tighter bound of jerk at the deceleration phase but a larger peak jerk at the acceleration phase. The motion profile design methodology is introduced, and a simulation study on the effects of motion induced stage vibration is also presented.

\section{Motion Profile Design Methodology}

The proposed motion profile is referred to as asymmetric super s-curve in this study. To minimize the change rate of the acceleration (jerk), the acceleration profile of a conventional S-curve is modified to have also an $\mathrm{S}$ shape for the acceleration and deceleration periods. A schematic view of the proposed acceleration, velocity, and displacement profiles is shown in Figure 1. This motion profile comprises three main parts: acceleration phase in $\left[t_{0}, t_{3}\right]$, constant velocity phase in $\left[t_{3}, t_{4}\right]$ and deceleration phase in $\left[t_{4}, t_{7}\right]$. For a point-to-point motion planning, it is more important to minimize the residual vibration when the motion stage reaches the target position. Considering such a characteristics, the acceleration phase can be designed to be more aggressive, while the deceleration phase to be smoother. It thus results in an asymmetric motion profile. The acceleration phase profile and deceleration phase profile can be further divided into three stages for each, so the whole process includes seven stages:

Stage 1: $\left[t_{0}, t_{1}\right]$ Acceleration ramps up from 0 to maximum acceleration $A_{i}$ with maximum jerk $J_{\alpha}$ in a time interval of $T_{1}=t_{1}-t_{2}$

Stage 2: $\left[t_{1}, t_{2}\right]$ Acceleration keeps unchanged at its maximum value $A_{i}$ in a time interval of $T_{2}=t_{2}-t_{1}$.

Stage 3: $\left[t_{2}, t_{3}\right]$ Acceleration ramps down from $A_{i}$ to 0 with maximum jerk $J_{\beta}$, in a time interval of $T_{3}=t_{3}-$ $t_{2}$. 
Stage 4: $\left[t_{3}, t_{4}\right]$ Velocity keeps unchanged at its maximum value $V$ in a time interval of $T_{4}=t_{4}-t_{3}$.

Stage 5: $\left[t_{4}, t_{5}\right]$ Deceleration ramps up from 0 to maximum deceleration $A_{i i}$ with maximum jerk $J_{\gamma}$, in a time interval of $T_{5}=t_{5}-t_{4}$.

Stage 6: $\left[t_{5}, t_{6}\right]$ Deceleration keeps unchanged at its maximum value $A_{i i}$ in a time interval of $T_{6}=t_{6}-t_{5}$.

Stage 7: $\left[t_{6}, t_{7}\right]$ Deceleration ramps down from $A_{i i}$ to 0 with maximum jerk $J_{\delta}$ in a time interval of $T_{7}=t_{7}-$ $t_{6}$.

Figure 1. Schematic view of the proposed motion profile
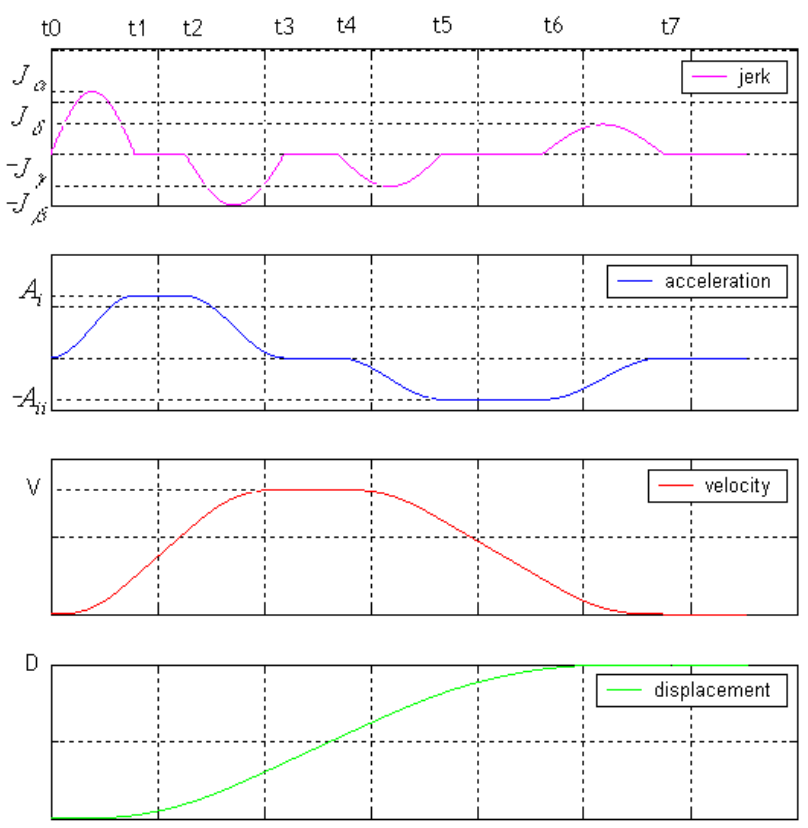

Suppose that a mass $m$ is designed to move a distance of $D$ with maximum velocity $V$, maximum acceleration $A_{i}$ during the accelerating phase at $\left[\mathrm{t}_{0}, \mathrm{t}_{3}\right]$, and a maximum deceleration $A_{i i}$ during the decelerating phase at $\left[\mathrm{t}_{4}, \mathrm{t}_{7}\right]$. The jerk constraints are set as $J_{\alpha}, J_{\beta}, J_{\gamma}, J_{\delta}$ in stage $1,3,5,7$ respectively. The displacement values at the end of each stage, i.e. $t_{1}, t_{2}, \ldots, t_{7}$, are designated as $d_{1}, d_{2}, \ldots, d_{7}$. From the boundary parameters and their mathematical relationships, the time interval of each stage in the motion can be determined by:

$T_{1}=\frac{\pi A_{i}}{2 J_{\alpha}}$

$T_{3}=\frac{\pi A_{i}}{2 J_{\beta}}$

$T_{5}=\frac{\pi A_{i i}}{2 J_{\gamma}}$

$T_{7}=\frac{\pi A_{i i}}{2 J_{\delta}}$

$T_{2}=\frac{V}{A_{i}}-\frac{T_{1}}{2}-\frac{T_{3}}{2}$
$T_{6}=\frac{V}{A_{i i}}-\frac{T_{5}}{2}-\frac{T_{7}}{2}$

$T_{4}=\frac{1}{V}\left(D-D_{i}-D_{i i}\right)$

Where $D_{i}$ and $D_{i i}$ are the travel distance within $\left[t_{0}, t_{3}\right]$ and $\left[t_{4}, t_{7}\right]$ respectively, which can be expressed as

$D_{i}=A_{i}\left(\frac{1}{4}-\frac{1}{\pi^{2}}\right)\left(T_{1}^{2}-T_{3}^{2}\right)+\frac{A_{i} T_{2}}{4}\left(T_{1}-T_{3}\right)+V\left(T_{3}+\frac{T_{2}}{2}\right)$

$D_{i i}=A_{i i}\left(\frac{1}{4}-\frac{1}{\pi^{2}}\right)\left(T_{7}^{2}-T_{5}^{2}\right)+\frac{A_{i i} T_{6}}{4}\left(T_{7}-T_{5}\right)+V\left(T_{7}+\frac{T_{6}}{2}\right)$

The total travel time $T$ is a sum of the time intervals of all the 7 stages:

$T=\sum_{i=1}^{7} T_{i}$

For the time intervals indicated in Eqs. (1) to (7), the instantaneous values of jerk, acceleration, velocity and displacement can be expressed as

For $t \in\left[t_{0}, t_{1}\right]$ :

$$
\begin{aligned}
& j(t)=J_{\alpha} \sin \frac{\pi}{T_{1}} t \\
& a(t)=\frac{A_{i}}{2}\left(1-\cos \frac{\pi}{T_{1}} t\right) \\
& v(t)=\frac{A_{i}}{2} t-\frac{A_{i} T_{1}}{2 \pi} \sin \frac{\pi}{T_{1}} t \\
& d(t)=\frac{A_{i}}{4} t^{2}-\frac{A_{i} T_{1}^{2}}{2 \pi^{2}}+\frac{A_{i} T_{1}^{2}}{2 \pi^{2}} \cos \frac{\pi}{T_{1}} t \\
& d_{1}=A_{i} T_{1}^{2}\left(\frac{1}{4}-\frac{1}{\pi^{2}}\right)
\end{aligned}
$$

For $t \in\left[t_{1}, t_{2}\right]$ :

$$
\begin{aligned}
& j(t)=0 \\
& a(t)=A_{i} \\
& v(t)=\frac{A_{i}}{2} T_{1}+A_{i}\left(t-t_{1}\right) \\
& d(t)=A_{i} T_{1}^{2}\left(\frac{1}{4}-\frac{1}{\pi^{2}}\right)+\frac{A_{i}}{2} T_{1}\left(t-t_{1}\right)+\frac{A_{i}}{2}\left(t-t_{1}\right)^{2} \\
& d_{2}=A_{i} T_{1}^{2}\left(\frac{1}{4}-\frac{1}{\pi^{2}}\right)+\frac{A_{i}}{2} T_{1} T_{2}+\frac{A_{i}}{2} T_{2}{ }^{2}
\end{aligned}
$$

For $t \in\left[t_{2}, t_{3}\right]$ :

$$
\begin{aligned}
& j(t)=-J_{\beta} \sin \frac{\pi}{T_{3}}\left(t-t_{2}\right) \\
& a(t)=\frac{A_{i}}{2}\left(1+\cos \frac{\pi}{T_{3}}\left(t-t_{2}\right)\right) \\
& v(t)=A_{i}\left(\frac{T_{1}}{2}+T_{2}\right)+\frac{A_{i}}{2}\left(t-t_{2}\right)+\frac{A_{i} T_{3}}{2 \pi} \sin \frac{\pi}{T_{3}}\left(t-t_{2}\right)
\end{aligned}
$$




$$
\begin{aligned}
& d(t)=A_{i} T_{1}^{2}\left(\frac{1}{4}-\frac{1}{\pi^{2}}\right)+\frac{A_{i}}{2} T_{1} T_{2}+\frac{A_{i}}{2} T_{2}^{2}+A_{i}\left(\frac{T_{1}}{2}+T_{2}\right)\left(t-t_{2}\right) \\
& +\frac{A_{i}}{4}\left(t-t_{2}\right)^{2}+\frac{A T_{3}^{2}}{2 \pi^{2}}-\frac{A T_{3}^{2}}{2 \pi^{2}} \cos \frac{\pi}{T_{3}}\left(t-t_{2}\right) \\
& d_{3}=A_{i}\left(\frac{1}{4}-\frac{1}{\pi^{2}}\right)\left(T_{1}^{2}-T_{3}^{2}\right)+\frac{A_{i} T_{2}}{4}\left(T_{1}-T_{3}\right)+V\left(T_{3}+\frac{T_{2}}{2}\right)=\mathrm{D}_{\mathrm{i}}
\end{aligned}
$$

For $t \in\left[t_{3}, t_{4}\right]$ :

$$
\begin{aligned}
& j(t)=0 \\
& a(t)=0 \\
& v(t)=A_{i}\left(\frac{T_{1}}{2}+T_{2}+\frac{T_{3}}{2}\right)=V \\
& d(t)=D_{i}+V\left(t-t_{3}\right) \\
& d_{4}=D_{1}+V T_{4}
\end{aligned}
$$

For $t \in\left[t_{4}, t_{5}\right]$ :

$$
\begin{aligned}
j(t) & =-J_{\gamma} \sin \frac{\pi}{T_{5}}\left(t-t_{4}\right) \\
a(t) & =-\frac{A_{i i}}{2}\left(1-\cos \frac{\pi}{T_{5}}\left(t-t_{4}\right)\right) \\
v(t) & =V-\frac{A_{i i}}{2}\left(t-t_{4}\right)+\frac{A_{i i} T_{5}}{2 \pi} \sin \frac{\pi}{T_{5}}\left(t-t_{4}\right) \\
d(t) & =D_{i}+V T_{v}+V\left(t-t_{4}\right)-\frac{A_{i i}}{4}\left(t-t_{4}\right)^{2} \\
& +\frac{A_{i i} T_{5}^{2}}{2 \pi^{2}}-\frac{A_{i i} T_{5}^{2}}{2 \pi^{2}} \cos \frac{\pi}{T_{5}}\left(t-t_{4}\right) \\
d_{5}= & D_{1}+V T_{4}+V T_{5}-A_{i i} T_{5}^{2}\left(\frac{1}{4}-\frac{1}{\pi^{2}}\right)
\end{aligned}
$$

For $t \in\left[t_{5}, t_{6}\right]$ :

$$
\begin{aligned}
j(t)= & 0 \\
a(t)= & -A_{i i} \\
v(t)= & V-\frac{A_{i i}}{2} T_{5}-A_{i i}\left(t-t_{5}\right) \\
d(t)= & D_{1}+V T_{4}+V T_{5}-A_{i i} T_{5}^{2}\left(\frac{1}{4}-\frac{1}{\pi^{2}}\right)+V\left(t-t_{5}\right) \\
& -\frac{A_{i i}}{2} T_{5}\left(t-t_{5}\right)-\frac{A_{i i}}{2}\left(t-t_{5}\right)^{2} \\
d_{6}= & D_{i}+V T_{4}+V\left(T_{5}+\frac{T_{6}}{2}\right)-A_{i i} T_{5}^{2}\left(\frac{1}{4}-\frac{1}{\pi^{2}}\right)+\frac{A_{i i} T_{6}}{4}\left(T_{7}-T_{5}\right)
\end{aligned}
$$

For $t \in\left[t_{6}, t_{7}\right]$ :

$$
\begin{aligned}
& j(t)=J_{\delta} \sin \frac{\pi}{T_{7}}\left(t-t_{6}\right) \\
& a(t)=-\frac{A_{i i}}{2}\left(1+\cos \frac{\pi}{T_{7}}\left(t-t_{6}\right)\right)
\end{aligned}
$$

$$
\begin{aligned}
v(t) & =V-\frac{A_{i i}}{2} T_{5}-A_{i i} T_{6}-\frac{A_{i i}}{2}\left(t-t_{6}\right)-\frac{A_{i i} T_{7}}{2 \pi} \sin \frac{\pi}{T_{7}}\left(t-t_{6}\right) \\
d(t) & =D_{i}+V T_{4}+V\left(T_{5}+\frac{T_{6}}{2}\right)-A_{i i} T_{5}^{2}\left(\frac{1}{4}-\frac{1}{\pi^{2}}\right) \\
& +\frac{A_{i i} T_{6}}{4}\left(T_{7}-T_{5}\right)+\frac{A_{i i}}{2} T_{7}\left(t-t_{6}\right)-\frac{A_{i i}}{4}\left(t-t_{6}\right)^{2} \\
& -\frac{A_{i i} T_{7}^{2}}{2 \pi^{2}}+\frac{A_{i i} T_{7}^{2}}{2 \pi^{2}} \cos \frac{\pi}{T_{7}}\left(t-t_{6}\right) \\
d_{7} & =D_{i}+V T_{4}+V\left(T_{5}+\frac{T_{6}}{2}\right)-A_{i i} T_{5}^{2}\left(\frac{1}{4}-\frac{1}{\pi^{2}}\right) \\
& +\frac{A_{i i} T_{6}}{4}\left(T_{7}-T_{5}\right)+A_{i i} T_{7}^{2}\left(\frac{1}{4}-\frac{1}{\pi^{2}}\right) \\
& =D_{i}+V T_{4}+D_{i i}
\end{aligned}
$$

When the peak acceleration values and jerk values at different phases are set as the same respectively, i.e., $A_{i}=$ $A_{i i}, J_{\alpha}=J_{\beta}=J_{\gamma}=J_{\delta}$, then the motion profile is symmetrical, which is a special case of the asymmetrical profile. To illustrate the effectiveness of the proposed asymmetrical profile, a simplified planning approach is used in this study. For a specified moving distance of $D$, with a maximum velocity $V_{\max }$ fixed, a peak acceleration $A_{\max }$ is used for both the accelerating phase and the decelerating phase. The maximum jerk at the acceleration phase is set to be larger than that in the deceleration phase, i.e., $J_{1}=\lambda J_{2}$, where $J_{1}=J_{\alpha}=J_{\beta}, J_{2}=J_{\gamma}=J_{\delta}$ and $\lambda \geq 1$.

\section{Simulation Study}

To assess the efficiency of the proposed motion profile, a series of simulation study has been carried out by using MATLAB. The dynamic model of a simplified single axis motion stage is shown in Fig. 2. It includes a flexible base structure with a weight of $m_{1}$, holding a motor to move a mass with an equivalent weight of $m_{2}$. The friction force between the moving mass and the machine base is ignored. The dynamics of the flexible base is assumed to be a singledegree-of-freedom mass-spring-damper system, with a spring stiffness $k$, and damping coefficient $c$.

Figure 2. Dynamic model of a motion stage with a moving mass and flexible supporting frame

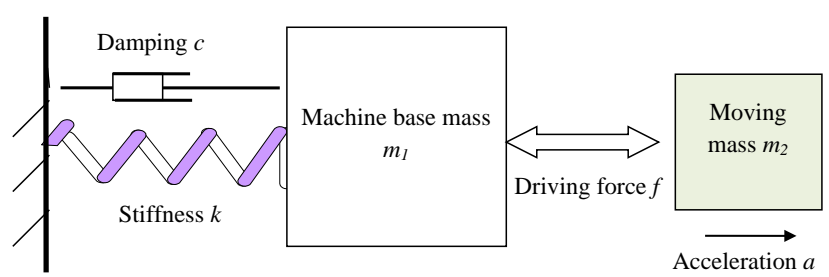

When the moving mass $m_{2}$ is driven with an acceleration $a$, the reaction force on the base structure $m_{1}$ will be

$$
F=m_{2} a
$$

The dynamics of the base structure can be expressed as

$m_{1} \ddot{x}+c \dot{x}+k x=F=m_{2} a$

Or in another form 
$\ddot{x}+2 \zeta \omega_{n} \dot{x}+\omega_{n}{ }^{2} x=\frac{m_{2}}{m_{1}} a$

where $\omega_{n}$ is the angular natural frequency of the base structure, $\zeta$ is the damping ratio, and $x$ is the vibration response of the base structure.

To carry out the simulation study, it is assumed that the ratio of the moving mass over the machine base is 0.1 , the undamped natural frequency of the base is $24 \mathrm{~Hz}$, with a damping ratio of $3 \%$, i.e., $m_{2} / m_{1}=0.1, \omega_{n}=150.8 \mathrm{rad} / \mathrm{s}$ (i.e., $f_{n}=24 \mathrm{~Hz}$ ), and $\zeta=3 \%$.

For comparison, three different motion profiles are evaluated, including the trapezoidal profile, s-curve profile, and the proposed super s-curve. At each simulation time step, the corresponding acceleration, velocity, and displacement values of the super s-curve motion profile can be obtained by using Eqs. (11-45). The residual vibration of the base frame is then determined by considering the instantaneous acceleration value and solving Eq. (48) to get the stage vibration. The MATLAB/SIMULINK model of the dynamic response of the base frame is shown in Fig. 3.

Figure 3. Simulation model for the motion induced stage vibration

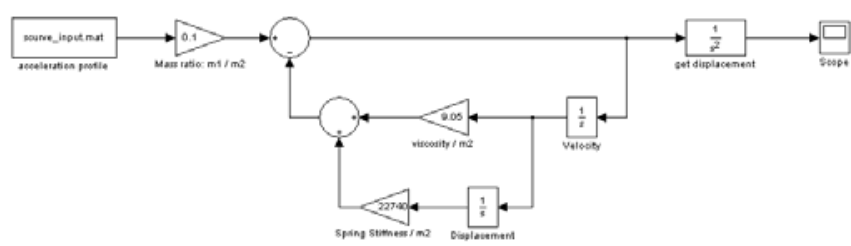

Figure 4. Comparisons of the acceleration from the trapezoidal, s-curve, and symmetric super s-curve motion profiles
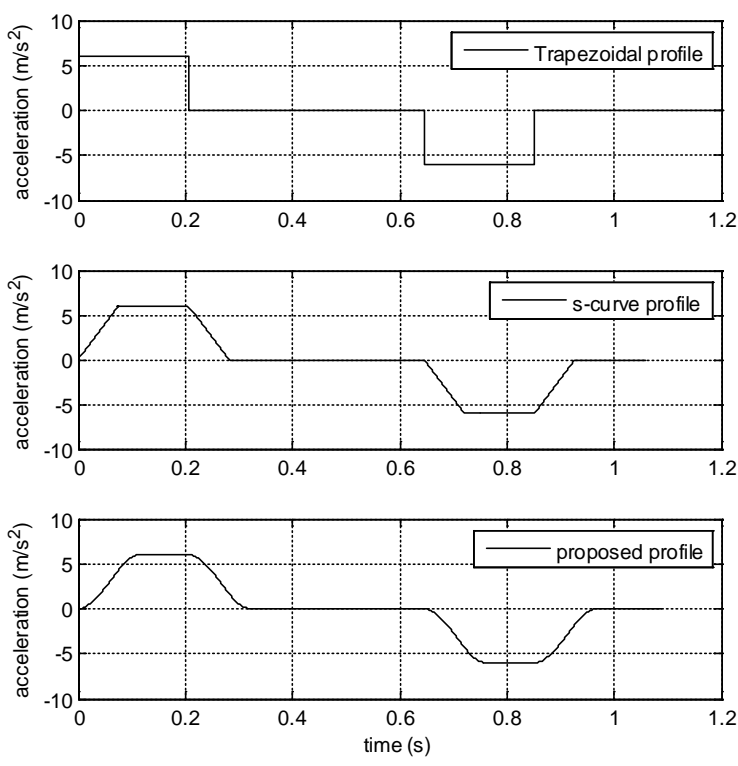

A symmetric super s-curve motion profile is used in the first set of simulation. The maximum velocity was set as 1.24 $\mathrm{m} / \mathrm{s}$ for all the three profiles, a maximum acceleration of 6 $\mathrm{m} / \mathrm{s}^{2}$ for the s-curve profile, and a maximum jerk of $80 \mathrm{~m} / \mathrm{s}^{3}$ for the symmetric super s-curve profile. The travel length was $0.8 \mathrm{~m}$. The travel time for different profiles was different, which was $0.852 \mathrm{sec}$ for the trapezoidal profile, $0.927 \mathrm{sec}$ for s-curve profile, and $1.006 \mathrm{sec}$ for the symmetric supers-curve.

The simulated motion acceleration profiles of the moving mass are shown in Fig. 4, and the vibration of the base structure excited by the stage motion using the three profiles are shown in Fig. 5. It is clear that the residual vibration excited by the trapezoidal profile is the worst, with a peakto-peak (PTP) value of about $50 \mu \mathrm{m}$. For the s-curve profile, the residual vibration has a PTP value of $9 \mu \mathrm{m}$, which is 5 times better than the trapezoidal profile. The super s-curve profile shows the best performance with a minimized vibration of about $1 \mu \mathrm{m}$ PTP, which is nearly 10 times better than the conventional s-curve profile, and the settling time is therefore significantly reduced.

Figure 5. Comparisons of the motion induced stage vibration from the trapezoidal, s-curve, and symmetric super s-curve motion profiles
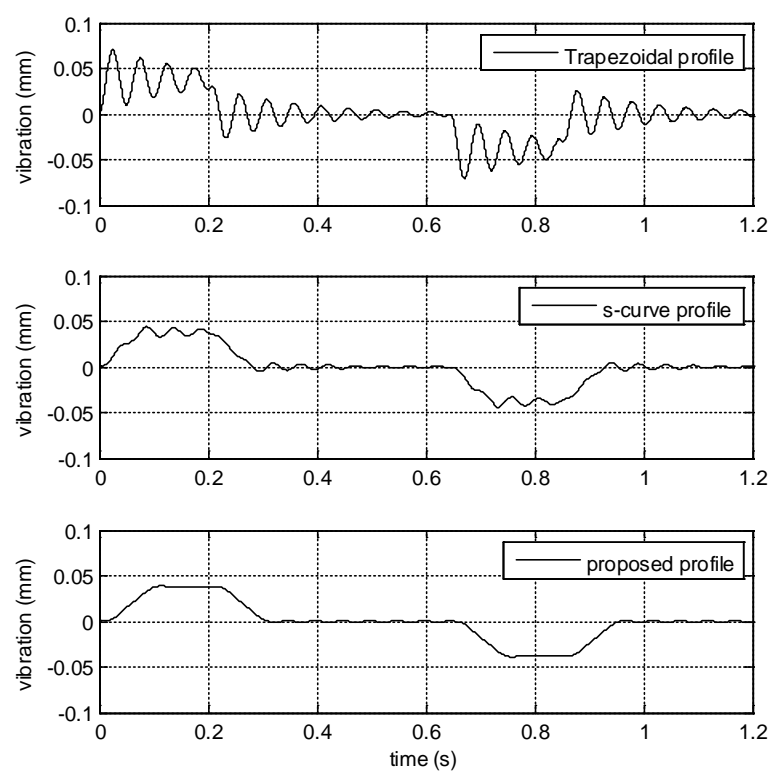

However, it is noted that a penalty for a smoother motion profile is a longer travel time under a set of fixed maximum acceleration and velocity values. The maximum acceleration and velocity are usually limited by the physical system such as motor power rate; moving mass, slide friction and damping, etc. In view of the fact that for most situations, the actual acceleration and velocity are far below the physical limitations, it is possible to design the smoother motion profile to be faster by using a higher allowable acceleration and jerk. The concern here is that the acceleration and jerk used are still under the physical limitation, and the induced structural vibration is under an acceptable level. Implementation of smooth motion profiles based on the level-shifted sinusoidal waveform by using a DSP motion controller has been demonstrated in (Li et al, 2005) and ( $\mathrm{Li}$ et al, 2009). For symmetric motion profile using the proposed jerk-constraint approach, the simulation results 
and experimental verification have been presented in ( $\mathrm{Li}$ et al, 2009). It has been shown that in comparison with conventional trapezoidal profile and s-curve profile, the motion induced stage vibration by using the super s-curve motion profile can be significantly reduced.

For the point-to-point motion, the positioning accuracy and settling time at the target point are more important. Thus, rather than to use a larger and identical peak acceleration and peak jerk for both the accelerating and decelerating phases in the symmetrical motion profile to achieve a same total move time as the trapezoidal profile, an asymmetric motion profile can be used with a larger peak jerk and acceleration at the acceleration phase, but with a smaller peak jerk and deceleration at the deceleration phase. In such a profile, it is expected that the resultant structural vibration at the end of the motion can be significantly reduced for a specific move time.

Figure 6. Comparisons of the jerk from the symmetric and asymmetric motion profiles

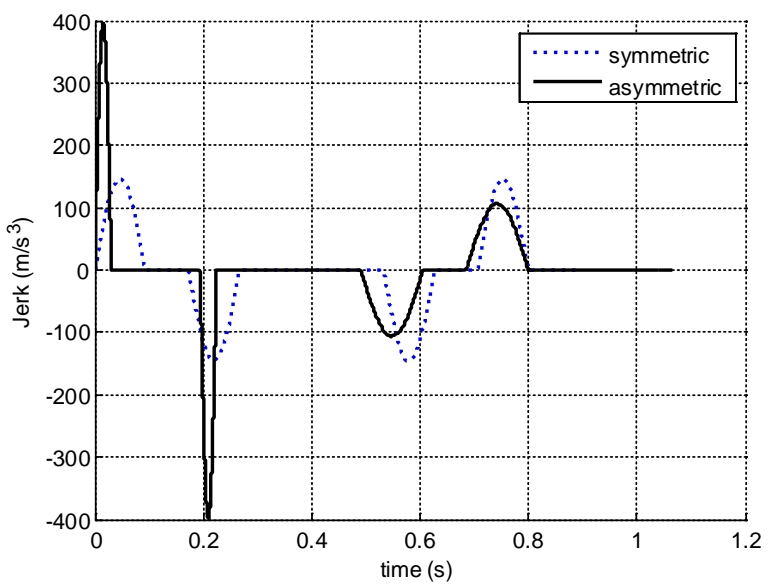

Figure 7. Comparisons of the acceleration from the symmetric and asymmetric motion profiles

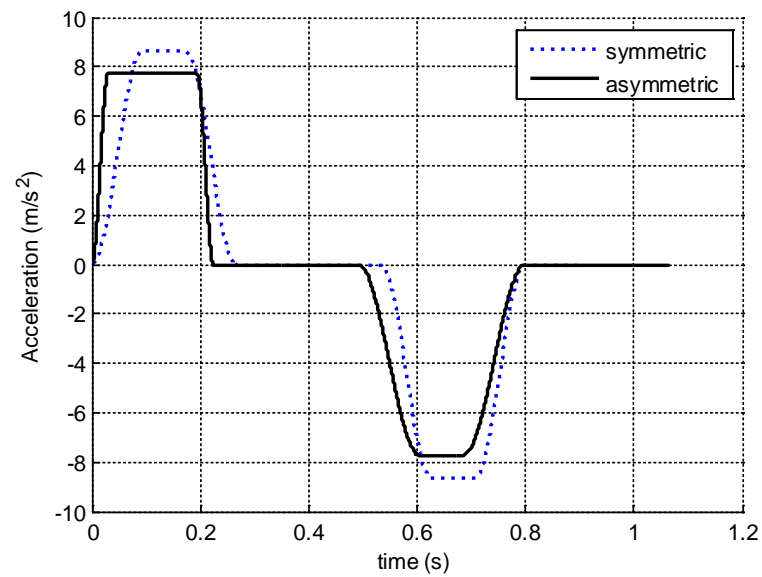

To demonstrate the efficiency of the asymmetric super scurve profile, a further simulation study was carried out to compare the performance of the symmetric and asymmetric profile. The motion time was fixed as $0.8 \mathrm{sec}$. The travel length was $0.8 \mathrm{~m}$, to be completed with a travel time of 0.8 sec. For the symmetric super s-curve profile, the peak jerk was set as $145.8 \mathrm{~m} / \mathrm{s}^{3}$, and the peak acceleration was 8.64 $\mathrm{m} / \mathrm{s}^{2}$. The peak velocity was $1.5 \mathrm{~m} / \mathrm{s}$. For the asymmetric profile, the peak jerk at the acceleration phase was 397.6 $\mathrm{m} / \mathrm{s}^{3}$, and a smaller peak jerk at the deceleration phase was used, which was $106.0 \mathrm{~m} / \mathrm{s}^{3}$. The peak acceleration was 7.72 $\mathrm{m} / \mathrm{s}^{2}$, for both the acceleration phase and the deceleration phase. Comparisons of the jerk, acceleration, velocity, and displacement of the symmetric and asymmetric profiles are shown in Figs. 6-9 respectively. It can be seen that for the asymmetric motion profile, it ramped up rapidly at the acceleration phase, but ramped down slowly at the deceleration phase with a much smoother change. A comparison of the motion induced stage vibration is illustrated in Fig. 10. It is clear that although the asymmetric motion profile resulted a larger stage vibration at the acceleration phase, which was caused by the larger jerk employed for rapid ramping up, the residual vibration at the target position was significantly reduced, which demonstrated an important characteristics of the proposed profile suitable for high speed point-to-point motion. It is expected that by fine tuning the jerk and acceleration values at different stages, the vibration can be further reduced. The profile can be implemented on a DSP-based motion controller in a similar way as presented in ( $\mathrm{Li}$ et al, 2009).

Figure 8. Comparisons of the velocity from the symmetric and asymmetric motion profiles

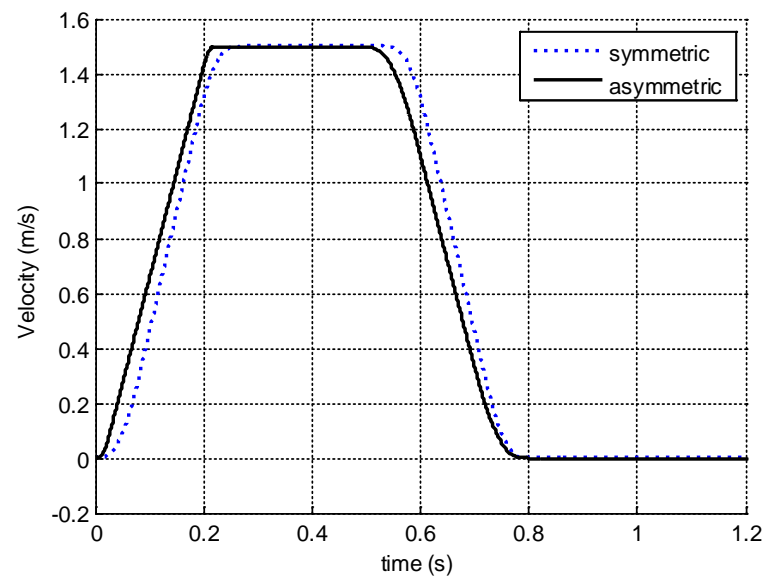

Figure 9. Comparisons of the motion displacement from the symmetric and asymmetric motion profiles

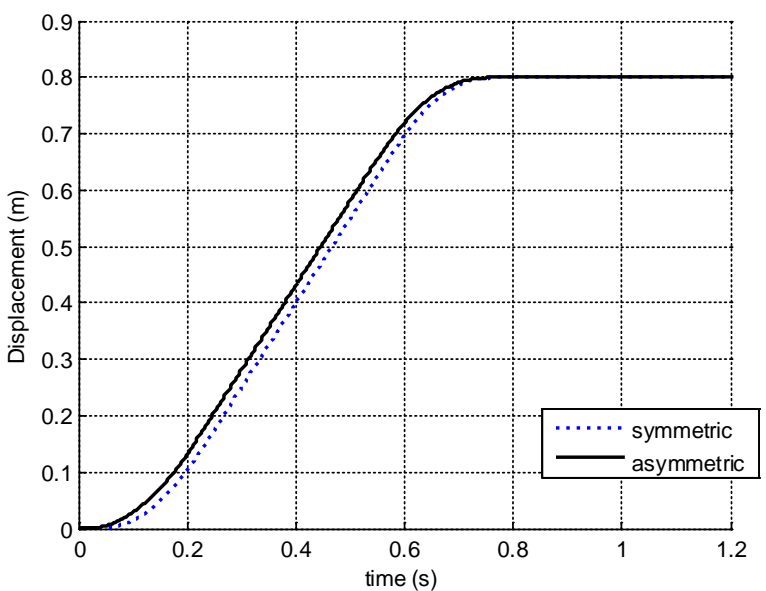


Figure 10. Comparisons of the motion induced stage vibration from the symmetric and asymmetric motion profiles

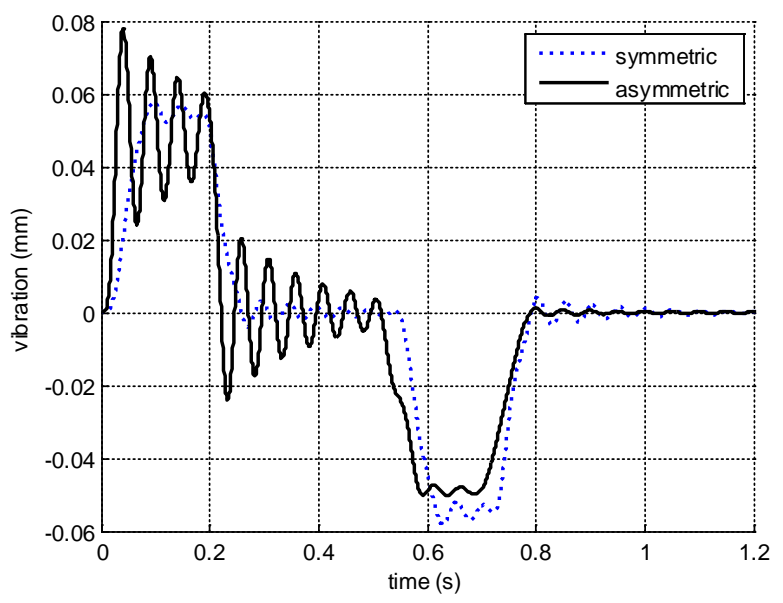

\section{Conclusions}

In this paper, a motion profile generation methodology is presented for reducing the motion induced residual vibrations for high-speed motion stages. In this method, the acceleration profile is smoothened by using sinusoidal functions. To enable the motion distance and motion time to be satisfied simultaneously, and also to further reduce the vibration, the jerk is more limited at the deceleration stage, and the deceleration bound is reduced as well, while a higher jerk is used at the acceleration phase. It results in an asymmetrical motion profile. The acceleration profile is used to derive the velocity and displacement profiles, which can be directly deployed in a DSP-based motion controller. A simulation study of the motion induced stage vibration has been conducted. By using this motion profile, the residual vibration as well as settling time can be greatly reduced compared to traditional profiles such as trapezoidal or S-curve profiles. The asymmetric profile also excels the symmetric profile in terms of the capability of faster rampup and further reduced residual vibration for high-speed point-to-point motion.

\section{References}

Bakar, S. A. A., Jamaluddin, H., Rahman, R. A., Samin, P. M., Masuda, R., Hashimoto, H., and Inaba, T. (2011). 'Modelling of magnetorheological semi-active suspension system controlled by semi-active damping force estimator'. International Journal of Computer Applications in Technology, vol. 42(1), pp. 49-64.

Butler, H. (2011) 'Position Control in Lithographic Equipment’. IEEE control systems. Volume:31 Issue:5. pp.28-47.

Ha, C., Rew, K., and Kim, K. (2008) 'A complete solution to asymmetric S-curve motion profile: Theory \& experiments', International Conference on Control, Automation and Systems, 2008. (ICCAS 2008). pp.2845 $-2849$.

Ha, C., Rew, K., and Kim, K. (2011) 'Formulating a Laplace domain approach for tuning motion profiles', 2011 IEEE/ASME International Conference on
Advanced Intelligent Mechatronics (AIM), pp. 356 360.

Hara, S. (2011) 'Positioning Control of Vibration Systems by Means of Simple Feedforward Control and Standard LQ Optimal Regulator', Third International Conference on Measuring Technology and Mechatronics Automation (ICMTMA), 2011 Vol. 2, pp. 877 - 882.

Lambrechts, P., Boerlage, M., and Steinbuch, M. (2005) 'Trajectory planning and feedforward design for electromechanical motion systems', Control Engineering Practice. Vol. 13, pp. 145-157.

Li, H.Z., Gong, Z., Lin, W., Jiang, T.Y., and Chen, X.Q. (2005) 'DSP-Based Motion Control of a NonCommutated DC Linear Motor Module', International Journal of Software Engineering and Knowledge Engineering. Vol. 15, No. 2. pp. 313-318.

Li, H.Z., Le, M.D., Gong, Z.M., and Lin, W., (2009) 'Motion Profile Design to Reduce Residual Vibration of High-speed Positioning Stages', IEEE/ASME Transactions on Mechatronics. Vol. 14/2. Pp. 264-269.

Li, T., Liu, Y., and Sun, L. (2012) 'Asymmetric Velocity Profile with Fixed Motion Time for Motion Control', Advanced Science Letters, Vol. 6, pp. 593-598.

Liu, Y., Liu, J., Li, L., and Pan, Y. (2011) 'Design and evaluation of a vibration sens or for measurement-whiledrilling'. International Journal of Computer Applications in Technology, Vol. 40(1), pp. 79-84.

Macfarlane, S., and Croft, E.A., (2003) 'Jerk-bounded manipulator trajectory planning: design for real-time applications', IEEE Transactions on Robotics and Automation, Volume 19, Issue 1. Pp.42 - 52.

Mahmood, I. A., Moheimani, S. O. R., and Bhikkaji, B. (2008) 'Precise tip positioning of a flexible manipulator using resonant control', IEEE/ASME Trans. Mechatronics, vol. 13, no. 2, pp. 180-186.

Rew, K., Ha, C., and Kim, K. (2009) 'A practically efficient method for motion control based on asymmetric velocity profile', International Journal of Machine Tools and Manufacture, Vol. 49, Issues 7-8, pp. 678682.

Rew, K., and Kim, K. (2010). 'A Closed-Form Solution to Asymmetric Motion Profile Allowing Acceleration Manipulation', IEEE Transactions on Industrial Electronics, Vol. 57 (7), pp. 2499 - 2506.

Wu, J., Xiong, Z., Lee, K., and Ding, H. (2011) 'HighAcceleration Precision Point-to-Point Motion Control With Look-Ahead Properties', IEEE Transactions on Industrial Electronics. Vol. 58, Issue: 9, , pp. 4343 4352.

Zou, F., Qu, D., Wang, J., and Xu, F. (2011) 'Asymmetric trajectory planning for vacuum robot motion', 2011 IEEE International Conference on Robotics and Automation (ICRA), pp. 1 - 4 . 